\title{
Effects of excluded volume on the electrolyte distribution around a charged sphere
}

\author{
P Strating and F W Wiegel \\ Centre for Theoretical Physics, Department for Applied Physics, Twente University, PO Box \\ 217, Enschede $7500 \mathrm{AE}$, The Netherlands
}

Received 9 October 1992

\begin{abstract}
In this paper a recently developed method is used to calculate the main effects of excluded volume on the distribution of ions around a charged central sphere in thermal equilibrium. The authors find significant corrections to the results of the conventional GouyChapman theory when the electrostatic energy due to the charge of the sphere is large compared with the thermal energy. The concentration shows a distinct saturation effect, while at the surface of the sphere the known saturation of the potential is lifted. Furthermore, the effect of excluded volume is found to be strongly dominated by the excluded volume of ions with a charge opposite to the charge of the sphere.
\end{abstract}

\section{Introduction}

The distribution of counterions around a central charge in a solvent is a well-known topic in chemistry and physics. The Gouy-Chapman model of a diffuse layer of ions shielding the central charge, and its linearization according to Debye-Hückel, are among the more familiar results, see, for instance, [1] ch 4 .

In real physical systems the counterions are not point particles, hence it is necessary to study the influence of steric hindrance on the counterion distribution. Physically one expects that because of the steric hindrance the counterion concentration cannot exceed a saturation value of $a^{-3}$, where $a^{3}$ is the effectively blocked (excluded) volume of a single ion. One expects steric hindrance to occur in regions of high concentration, i.e. close to the central charge.

In a recent paper [2] the authors studied the effect of the excluded volume of the counterions on the charge distribution around the sphere, using a new method, which had recently been developed by one of the authors [3] and which provides a particularly simple way of dealing with the main effect of the excluded volume of more or less spherical particles. They found a nice effect of saturation in the region of high concentration near the central sphere. However, only the case in which there is only one species of counterions was considered, while in the usual Gouy-Chapman model the sphere is immersed in a neutral fluid containing two types of counterion with opposite charge. Experimentally this formulation of the problem is also important, as most ionic solutions are the result of dissolving a salt into positive and negative ions. To appreciate fully the corrections to the Gouy-Chapman results obtained with this new method, the authors now present a calculation taking into account the mobility of the second type of counterions. The method, however, requires that these additional counterions have the same effective excluded volume as the counterions of the first type. 
We will study the charge distribution around a central sphere of radius $R$ and total charge $Q$. The solvent, with dielectric constant $\varepsilon$, contains two types of counterion with spatial concentration $c_{+}(r)$ and $c_{-}(r)$. Each counterion carries a charge of $q$ or $-q$ (where we assume that $q Q<0$ ) and has an effective excluded volume $a^{3}$. At infinity the concentration of ions is $c_{+}(\infty)$ and $c_{-}(\infty)$, and to obtain neutrality we demand $c_{+}(\infty)=c_{-}(\infty) \equiv c_{0}$.

We want to calculate the counterion concentrations $c_{ \pm}(r)$ in thermal equilibrium. Because of the spherical symmetry the concentrations $c_{ \pm}$depend only on the radial distance $r$ from the centre of the sphere.

\section{Basic equations}

In the method described in [3] the excluded volume is taken into account by adding to the diffusive current density, which is usually given by $j_{\mathrm{D}, \pm}(r)=-D_{0} \nabla c_{ \pm}(r)$, an extra term describing the steric hindrance

$$
j_{D, \pm}=-D_{0} \nabla c_{ \pm}-\frac{D_{0} a^{3} c_{ \pm}}{1-a^{3}\left(c_{+}+c_{-}\right)} \nabla\left(c_{+}+c_{-}\right)
$$

where $D_{0}$ is the diffusion coefficient, given by $k_{\mathrm{B}} T / f_{\mathrm{T}}$ with $f_{\mathrm{T}}$ the translational coefficient of friction, $k_{\mathrm{B}}$ Boltzmann's constant and $T$ the temperature. Equation (1) is an example of gradient diffusion and can be obtained as follows.

If inertial effects are neglected, the particles move in the direction of a local average force $F_{2 v}$, which is assumed to be slowly varying. The particle current density becomes $j=c(r) v(r)$, with $c(r)$ the local concentration and $v(r)=F_{\mathrm{av}}(r) / f_{\mathrm{T}}$ the local average velocity. We now assume that this current density can be written as the sum of two terms:

$$
j=j_{\mathrm{D}}+j_{\mathrm{ext}}=j_{\mathrm{D}}-c(r) \nabla V_{\mathrm{ext}} / f_{\mathrm{T}}
$$

where $j_{D}$ is due to diffusion with steric hindrance and the second term is the result of an external force with potential $V_{\text {ext. }}$. In equilibrium, the total density current must be identically zero, yielding a relation between $j_{\mathrm{D}}$ and the external potential. Furthermore, in equilibrium a relation exists between $c(r)$ and the external potential, which can be used to eliminate the external potential. This relation, however, should contain the effects of excluded volume, because using the Boltzmann equilibrium distribution only yields the normal diffusion equation. An approximate equilibrium distribution can be found by treating the rigid particles as if they were placed on a lattice, and noticing that the excluded volume phenomenon is equivalent to a geometric exclusion principle. The equilibrium distribution is then found to be

$$
c(r)=a^{-3} /\left(1+e^{8 V_{e x t}(r)-\xi}\right)
$$

which is a Fermi-Dirac-like distribution ( $\xi$ is a constant Lagrange multiplier). Equation (3) can be readily extended to the case in which two types of counterion are present, from which equation (1) follows. The result no longer depends on the external potential and $\xi$. Note that a class of diffusion equations can be derived from knowledge of the equilibrium distributions for various systems, of which the lattice system is only one example.

We can now write down the basic formulae. The local particle current density is given by

$$
j_{ \pm}(r)=-D_{0} \nabla c_{ \pm}-\frac{D_{0} a^{3} c_{ \pm}}{1-a^{3}\left(c_{+}+c_{-}\right)} \nabla\left(c_{+}+c_{-}\right)+\frac{c_{ \pm} F_{ \pm}}{f_{T}}
$$


$F_{ \pm}$is the force on a single counterion of the kind + or - , given by $F_{ \pm}(r)= \pm q E(r)$ with $E(r)$ the local (average) electrostatic field. In thermal equilibrium the current of equation (4) should vanish identically; using spherical symmetry and $E=-\nabla \varphi$ ( $\varphi$ is the electrostatic potential), we find

$$
\pm \frac{q}{k_{\mathrm{B}} T} \frac{\mathrm{d} \varphi}{\mathrm{d} r}=-\frac{1}{c_{ \pm}} \frac{\mathrm{d} c_{ \pm}}{\mathrm{d} r}-\frac{a^{3}}{1-a^{3}\left(c_{+}+c_{-}\right)} \frac{\mathrm{d}}{\mathrm{d} r}\left(c_{+}+c_{-}\right) .
$$

These are two coupled equations relating $\varphi(r)$ to $c_{+}(r)$ and $c_{-}(r)$. To obtain a closed set of equations we need a third equation. We invoke the Maxwell relation div $\varepsilon E=4 \pi \rho$ (we use Gaussian units in which $\varepsilon_{0}=1$; see, for instance, [4]), which reads, in terms of the potential,

$$
\Delta \varphi=-4 \pi \rho / \varepsilon
$$

with the charge density $\rho(r)=q c_{+}(r)-q c_{-}(r)$ outside the sphere. Thus we have found a third relation between $\varphi$ and $c_{ \pm}$; in spherical coordinates

$$
\left(\frac{\mathrm{d}}{\mathrm{d} r}+\frac{2}{r}\right) \frac{\mathrm{d} \varphi}{\mathrm{d} r}=-\frac{4 \pi q}{\varepsilon}\left(c_{+}(r)-c_{-}(r)\right) .
$$

Before continuing we simplify the appearance of these formulae by introducing the dimensionless concentrations $f=a^{3} c$ and $f_{0}=a^{3} c_{0}$, which are effective volume fractions, and a dimensionless coordinate $x=\left(x_{0} / R\right) r$ with

$$
x_{0}=\frac{R}{a} \sqrt{\frac{4 \pi q^{2} / \varepsilon a}{k_{\mathrm{B}} T}} .
$$

We have written $x_{0}$ in this particular form to show that the only length scale in the problem is the result of two factors: a geometrical factor $R / a$ and a factor $\sqrt{\left(4 \pi q^{2} / \varepsilon a\right) / k_{\mathrm{B}} T}$, which measures the maximum energy of the electrostatic interaction of two ions outside the central sphere, compared with the thermal energy.

In terms of $f$ and $x$ equations (5) and (7) are (for $x \geqslant x_{0}$ )

$$
\left(\frac{\mathrm{d}}{\mathrm{d} x}+\frac{2}{x}\right) \frac{q}{k_{\mathrm{B}} T} \frac{\mathrm{d} \varphi}{\mathrm{d} x}=-\left(f_{+}(x)-f_{-}(x)\right)
$$

and

$$
\pm \frac{q}{k_{\mathrm{B}} T} \frac{\mathrm{d} \varphi}{\mathrm{d} x}=-\frac{1}{f_{ \pm}} \frac{\mathrm{d} f_{ \pm}}{\mathrm{d} x}-\frac{1}{1-\left(f_{+}+f_{-}\right)} \frac{\mathrm{d}}{\mathrm{d} x}\left(f_{+}+f_{-}\right) .
$$

One now wants to combine these three equations. To achieve this we introduce two functions

$$
g_{ \pm}=\ln \frac{f_{ \pm}}{1-f_{+}-f_{-}}-\ln \frac{f_{0}}{1-2 f_{0}} .
$$

The constant on the right-hand side has been added for later convenience. These relations can be inverted to

$$
f_{ \pm}=\frac{f_{0} \mathrm{e}^{g \pm}}{1-2 f_{0}+f_{0} \mathrm{e}^{g+}+f_{0} \mathrm{e}^{g-}} .
$$


Then the equations (10) can be rewritten as

$$
\frac{q}{k_{\mathrm{B}} T} \frac{\mathrm{d} \varphi}{\mathrm{d} x}=-\frac{\mathrm{d} g_{+}}{\mathrm{d} x}=+\frac{\mathrm{d} g_{-}}{\mathrm{d} x}
$$

with the solutions

$$
\frac{q \varphi}{k_{\mathrm{B}} T}=-g_{+}+A_{+}=+g_{-}+A_{-}
$$

where the $A_{ \pm}$are integration constants. Because of the neutrality of the fluid, at infinity $\varphi$ is supposed to be zero at infinity. Then, using the boundary condition for $f$, we find that the $g_{ \pm}$should also be zero at infinity. Therefore the constants $A_{ \pm}$must be zero and

$$
g_{+}(x)=-g_{-}(x)=-q \varphi / k_{\mathrm{B}} T .
$$

So $g_{ \pm}$are dimensionless potentials. We now call $g=g_{+}$and solve in terms of this function. This gives us our fundamental equation with only one unknown function

$$
\left(\frac{\mathrm{d}}{\mathrm{d} x}+\frac{2}{x}\right) \frac{\mathrm{d} g}{\mathrm{~d} x}=\frac{f_{0}\left(\mathrm{e}^{g}-\mathrm{e}^{-g}\right)}{1-2 f_{0}+f_{0} \mathrm{e}^{g}+f_{0} \mathrm{e}^{-g}} .
$$

From the solution $g(x)$ of this equation, $\varphi(x)$ and $f_{ \pm}(x)$ can be derived using equations (12) and (15).

Besides the boundary condition for $g$ at infinity we need to have a second boundary condition to incorporate the charge of the central sphere and to find a unique solution. Therefore we integrate equation (6) over the volume $V_{R}$ of the sphere with radius $R$ and use Gauss' theorem to obtain

$$
\int_{V_{R}} \Delta \varphi(r) \mathrm{d} r=\left.4 \pi R^{2} \frac{\mathrm{d} \varphi}{\mathrm{d} r}\right|_{r=R}=-\frac{4 \pi Q}{\varepsilon}
$$

which gives us the desired boundary condition

$$
\left.\frac{\mathrm{d} \varphi}{\mathrm{d} r}\right|_{r=R}=-\frac{Q}{\varepsilon R^{2}}
$$

or, in terms of $g$,

$$
\left.\frac{\mathrm{d} g}{\mathrm{~d} x}\right|_{x=x_{0}}=-\frac{\mu}{x_{0}}
$$

where we have introduced the dimensionless parameter $\mu=-q Q / k_{\mathrm{B}} T \varepsilon R$, which is always greater than zero and measures the competition between the thermal energy and the potential energy of the counterions at the surface of the sphere. Recalling equation (8) one sees that the solution of equation (16) depends on the various parameters in the system only through the three dimensionless parameters $R / a,\left(4 \pi q^{2} / \varepsilon a\right) / k_{\mathrm{B}} T$ and $q Q / k_{\mathrm{B}} T \varepsilon R$; more specifically through their combination $\mu / x_{0}$. 


\section{The Debye-Hückel limit}

In the Debye-Hückel theory one linearizes the nonlinear differential equation for $\varphi(r)$ (equation (16)) in the region where $q \varphi / k_{\mathrm{B}} T \ll 1$, which may be true everywhere outside the central sphere, but usually only holds in the asymptotic region $r \rightarrow \infty$. We discuss this linearization briefty.

Note that the quantity which should be small is our function $g$. After linearization of equation (16) we are left with

$$
\left(\frac{\mathrm{d}}{\mathrm{d} x}+\frac{2}{x}\right) \frac{\mathrm{d} g}{\mathrm{~d} x}=2 f_{0} g
$$

This equation can be solved exactly to give the asymptotic solution for $g$ (the $\sim$ sign denotes proportionality)

$$
g(x) \sim \frac{\mathrm{e}^{-\sqrt{2 f_{0}} x}}{x}, \quad \text { or } \quad g(r) \sim \frac{\mathrm{e}^{-\kappa r}}{r}
$$

with the Debye-Hückel inverse screening length $\kappa$

$$
\kappa=\frac{x_{0}}{R} \sqrt{f_{0}}=\sqrt{\frac{4 \pi q^{2} c_{0}}{k_{\mathrm{B}} T \varepsilon}} .
$$

This is precisely the same result as one would obtain neglecting excluded volume effects. The $a^{3}$-dependence has disappeared by inserting $f_{0}=a^{3} c_{0}$ into equation (22).

If the linearization is valid throughout the whole space, solution (21) should satisfy the boundary condition at $x_{0}$ as well, and one finds the approximate solution

$$
g(x) \approx \frac{\mu}{\left(1 / x_{0}\right)+\sqrt{2 f_{0}}} \frac{e^{-\sqrt{2 f_{0}}\left(x-x_{0}\right)}}{x}
$$

\section{Properties of the solution and numerical results}

Before turning to the results of a numerical integration of the full equation we give some general properties of the solution of equation (16). From a physical point of view, $g$ must be smooth and bounded for all $x>x_{0}$. We now show that $g$ is positive and monotonically decreasing. Suppose $\mathrm{d} g / \mathrm{d} x=0$ at $x=\bar{x}$. Then according to equation (16) this must always be a minimum if $g(\bar{x})>0$, because the second derivative of $g$ is positive. But to satisfy the boundary condition at infinity it would require an additional maximum, and a maximum is not possible as long as $g>0$. A similar argument shows that $g$ cannot have an extremum below the line $g=0$. Moreover, with the same reasoning, $g$ can never cross the line $g=0$. Finally, we are left to show that $g$ cannot reach the line $g=0$ with zero derivative at finite $\bar{x}$. Again, suppose this would happen, then $g$ would obey the linearized equation (20) in a small region near $\bar{x}$. Solving this equation shows that the only solution with the desired properties at $\bar{x}$ is $g(x)=0$ for all $x$ near $\bar{x}$, and this means that the region of validity of the linearization can be enlarged to find that $g=0$ in this larger region. Repeating this process, the region of linearization can be arbitrarily enlarged to comprise the whole range $x \geqslant x_{0}$. But the solution $\mathrm{g}=0$ for all $x$ does not satisfy the boundary condition at $x_{0}$ 


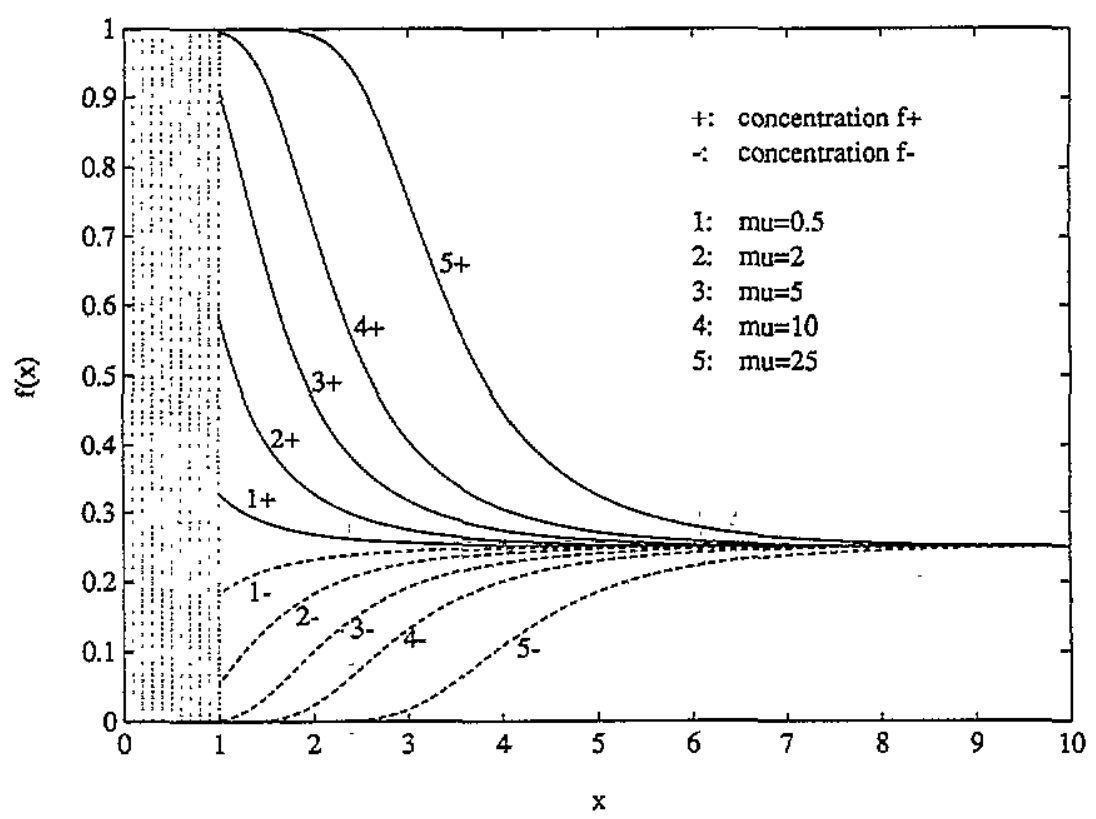

Figure 1. The concentrations $f_{+}$and $f_{-}$as a function of $x\left(x_{0}=1, f_{0}=0.25\right)$.

and we must discard the possibility that $g$ becomes zero at finite $x$. Because $g$ starts off at $x_{0}$ with a negative derivative, we can conclude that $g$ is a positive definite, monotonically decreasing function. With these properties of $g$, it is easily shown using equation (12) that $f_{+}$is monotonically decreasing and $f_{0}<f_{+}<1$, and that $f_{-}$is monotonically increasing and $0<f_{-}<f_{0}$.

In figure 1 we have plotted $f_{+}$and $f_{-}$, as calculated from the numerical solutions of equation (16), for various boundary conditions. The solutions $g$ were calculated using fourth- and fifth-order Runge-Kutta algorithms. It is clearly visible that while the solution for $f_{+}$is extremely unexciting for $\mu / x_{0} \leqslant 1$, it develops a beautiful plateau close to the sphere for larger ratios of $\mu / x_{0}$. So when the attractive force of the central sphere is strong as compared to the diffusive force, there is a region of saturation with close packing concentration $c_{+}=a^{-3}$. At the same time the counterions with opposite charge are expelled from this region. In figure 2 we have plotted the solutions $g(x)$ of equation (16) themselves.

Table 1 gives the values of the dimensionless potential at the surface of the sphere for various boundary conditions, i.e. for different $\mu / x_{0}$ and different $f_{0}$. The solutions $f$ and $g$ do not only depend on $\mu / x_{0}$, but also on $f_{0}$, the concentration at infinity, but only in a quantitative way. The qualitative behaviour is identical for all $f_{0}<1$. We only note that for higher values of $f_{0}$ the effect of excluded volume is visible for smaller $\mu$. Table 1 shows that $g\left(x_{0}\right)$ does not depend very strongly on $f_{0}$.

Curve $3(\mathrm{a})$ is a plot of the values of $g\left(x_{0}\right)$ at the surface of the sphere corresponding to figure 2 ; it can be seen that the potential at the surface depends almost linearly on the value of $\mu$. This is quite remarkable, because it is known that in the Gouy-Chapman model the potential saturates at the surface. The disappearance of the saturation of the potential is related directly to the fact that the particle concentration cannot exceed the close packing value $a^{-3}$. The slope of the curve 3 (a) for small $\mu$ can be calculated to be $\left(1+x_{0} \sqrt{2 f_{0}}\right)^{-1}$, using the approximate solution (23). To show the difference between our present results and the usual Gouy-Chapman model without excluded volume, curve $3(\mathrm{~b})$ is the plot of 


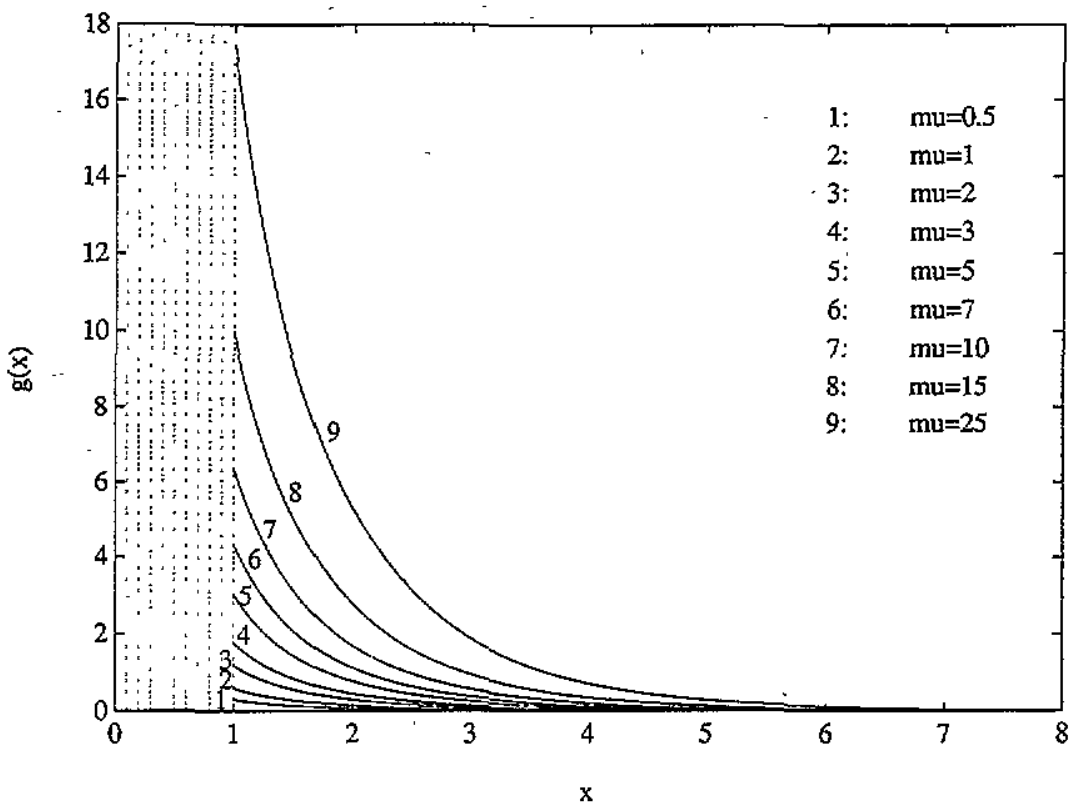

Figure 2. The dimensionless potential $g$ as a function of $x\left(x_{0}=1, f_{0}=0.25\right)$.

Table 1. Potential values at the surface of the sphere for different boundary conditions $\mu$ and $f_{0}\left(x_{0}=1\right)$.

\begin{tabular}{llllllll}
\hline \multicolumn{8}{c}{$f_{0}$} \\
\hline$\mu$ & 0.01 & 0.05 & 0.1 & 0.2 & 0.25 & 0.3 & 0.4 \\
\hline 0.1 & 0.0871 & 0.0760 & 0.0691 & 0.0613 & 0.0586 & 0.0564 & 0.0528 \\
0.5 & 0.436 & 0.380 & 0.345 & 0.306 & 0.293 & 0.282 & 0.264 \\
1 & 0.873 & 0.758 & 0.690 & 0.613 & 0.587 & 0.565 & 0.531 \\
2 & 1.73 & 1.51 & 1.38 & 1.23 & 1.18 & 1.14 & 1.08 \\
3 & 2.60 & 2.25 & 2.05 & 1.85 & 1.78 & 1.73 & 1.64 \\
5 & 4.24 & 3.68 & 3.40 & 3.12 & 3.03 & 2.96 & 2.85 \\
7 & 5.79 & 5.09 & 4.76 & 4.44 & 4.34 & 4.26 & 4.13 \\
10 & 8.01 & 7.22 & 6.86 & 6.50 & 6.39 & 6.29 & 6.15 \\
15 & 11.9 & 10.9 & 10.5 & 10.1 & 9.96 & 9.86 & 9.70 \\
20 & 15.7 & 14.7 & 14.2 & 13.8 & 13.7 & 13.6 & 13.4 \\
25 & 19.6 & 18.5 & 18.1 & 17.6 & 17.5 & 17.4 & 17.2 \\
\hline
\end{tabular}

the values of $g\left(x_{0}\right)$ obtained in the absence of excluded volume effects. At approximately $\mu=3$ curves $\mathrm{a}$ and $\mathrm{b}$ separate.

As we have noted in the introduction, the present method is only applicable if the two counterion species have the same excluded volume $a^{3}$. However, the concentration profile in figure 1 suggests that the physics of packing in this problem is dominated by only one type of counterion, namely those ions with charge opposite to the central charge. To get a qualitative picture of the effect of an asymmetry in the excluded volume of the two types of ion, we have done calculations for the two cases in which one type of ion has zero excluded volume and the other has excluded volume $a^{3}$. These are the extremal cases of the largest possible difference in excluded volume, for which it is also possible to write down closed 


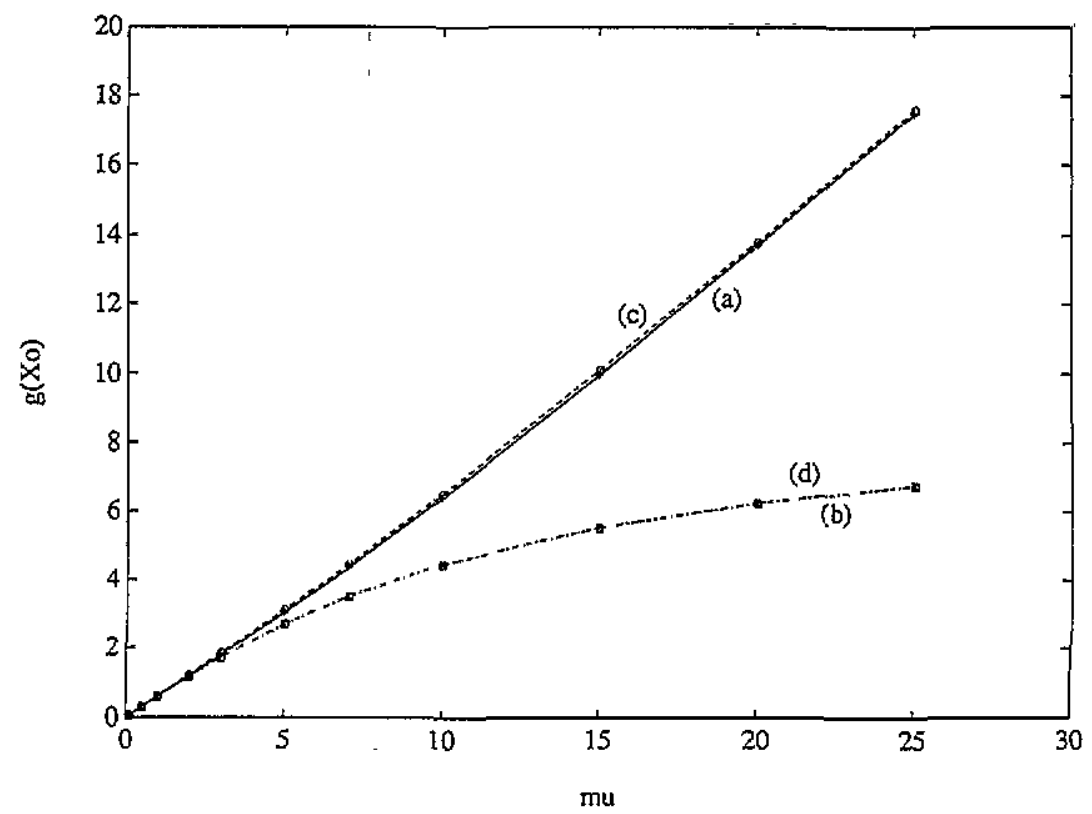

Figure 3. Potential values at the surface of the sphere $\left(x_{0}=1, f_{0}=0.25\right.$ ), with curve (a) the present method, both ion species have excluded volume $a^{3}$; curve (b) the Gouy-Chapman model, no excluded volume; curve (c), only $+q$ ions have non-zero excluded volume; and curve (d) only $-q$ ions have non-zero excluded volume.

equations for the dimensionless potential $g$. One finds

$$
\left(\frac{\mathrm{d}}{\mathrm{d} x}+\frac{2}{x}\right) \frac{\mathrm{d} g}{\mathrm{~d} x}=\frac{f_{0} \mathrm{e}^{8}}{1-f_{0}+f_{0} \mathrm{e}^{g}}-f_{0} \mathrm{e}^{-g}
$$

if the ions with charge $+q$ have non-zero excluded volume, and

$$
\left(\frac{\mathrm{d}}{\mathrm{d} x}+\frac{2}{x}\right) \frac{\mathrm{d} g}{\mathrm{~d} x}=f_{0} \mathrm{e}^{g}-\frac{f_{0} \mathrm{e}^{-g}}{1-f_{0}+f_{0} \mathrm{e}^{-g}}
$$

if the ions with charge $-q$ have non-zero excluded volume. These equations can be derived by assuming a Boltzmann equilibrium distribution for the ions without excluded volume and again a Fermi-Dirac-like distribution for the ions with excluded volume $a^{3}$. For these equations the potential values at the surface of the sphere have been calculated and plotted as respectively curves $3(\mathrm{c})$ and $3(\mathrm{~d})$. One sees that curve $3(\mathrm{c})$ almost coincides with curve 3(a), while curve 3(d) seems to coincide with 3(b) and cannot be seen at all, which is caused by the limited resolution of the graph. Furthermore, the shape of the curve $g(x)$ shows the same tendency to follow either the case of no excluded volume at all, or the case where both types of counterion have the same excluded volume. We can conclude that, in the region where excluded volume is important, only one type of counterions dominates the physics. This suggests that, to a very good approximation, our method may also be applied to problems with asymmetric excluded volume, as long as one takes as $a^{3}$ the effective excluded volume of the ions with charge opposite to the central charge, i.e. the $+q$ ions. In dynamical problems, however, the situation may be more complicated. 


\section{Conclusions}

In our previous paper [2] we have argued that with the present method it is possible to extend the Gouy-Chapman model into the so-called Stern layer. This is a layer of close packed, immobilized ions attached to the surface of a central charge. In this paper we have demonstrated that the same method is also applicable if there is more then one type of counterion present, which is not a priori obvious, and which increases the relevance of the calculations for real physical systems. Although the method is only applicable if the two counterion species have the same excluded volume $a^{3}$, we have argued that the results will not be very different if the counterions do not have the same excluded volume. This is caused by the fact that in the region where excluded volume plays a role, the ions with charge $-q$ are expelled, leaving the region effectively to the ions of opposite charge. This property greatly enhances the possibilities of testing the results.

One interpretation of equation (1) is that we have added a steric repulsion force to the diffusive force. This steric force is given by

$$
F_{\mathrm{st}}=k_{\mathrm{B}} T \nabla \ln \left(1-a^{3}\left(c_{+}+c_{-}\right)\right)
$$

If we combine this with the quite common expression for the diffusive, often called 'Brownian', force $F_{B}=-k_{\mathrm{B}} T \nabla \ln c / c_{0}$, one sees that the diffusion process with excluded volume is governed by a new Brownian force

$$
F_{ \pm}=-k_{\mathrm{B}} T \nabla \ln \frac{a^{3} c_{ \pm}}{1-a^{3}\left(c_{+}+c_{-}\right)}
$$

which is a strongly repulsive force in regions of close packing.

Finally, despite fierce nonlinearities, the physical solutions of equation (16) are smooth and well behaved (as well as the concentrations derived from them) and this fact is very encouraging if one wants to apply the method to various problems in physics.

\section{References}

[1] Russel W B, Saville D A and Schowalter W R 1989 Colloidal Dispersions (Cambridge: Cambridge. University Press)

[2] Strating P and Wiegel F W 1993 Distribution of ions around a charged sphere to be published

[3] Wiegel F W 1991 Physical Principles in Chemoreception (Lecture Notes in Biomathematics) (Berlin: Springer)

[4] Jackson J D 1975 Classical Electrodynamics 2nd edn (New York: Wiley) 